\title{
A Case Report of Two Primary Cancers, Breast Cancer with Adrenal Gland Metastatic and Second Primary Neuroendocrine Tumor in Colon, a Rare Case in Al-Bashir Hospital
}

\author{
Shaimaa Shamoun*, Ahmad Shamout \\ Clinical Oncology and Radiation Therapy Department, Al-Bashir Hospital, Al-Ashrafiyah, Amman, Jordan \\ Email: *shai_aviv@hotmail.com
}

How to cite this paper: Shamoun, S. and Shamout, A. (2018) A Case Report of Two Primary Cancers, Breast Cancer with Adrenal Gland Metastatic and Second Primary Neuroendocrine Tumor in Colon, a Rare Case in Al-Bashir Hospital. Case Reports in Clinical Medicine, 7, 306-313. https://doi.org/10.4236/crcm.2018.74028

Received: January 31, 2018

Accepted: April 24, 2018

Published: April 27, 2018

Copyright $\odot 2018$ by authors and Scientific Research Publishing Inc. This work is licensed under the Creative Commons Attribution International License (CC BY 4.0). http://creativecommons.org/licenses/by/4.0/

\section{Open Access}

\begin{abstract}
A 68 years old female, was diagnosed as a case of right breast cancer in 2013; grade II/III according to Bloom Richardson grading, Modify radical mastectomy (MRM) was done and the pathology report showed moderately differentiated invasive ductal carcinoma (IDC), stage T3N3M0. Immunohisrochemisty (IHC) findings revealed a tumor with Triple positive. Patient refused to treat by chemotherapy. Patient was given adjuvant trastuzumab (first dose $8 \mathrm{mg} / \mathrm{kg}$, followed by $6 \mathrm{mg} / \mathrm{kg}$ every three weeks) for the period of one year (16) cycles after which she went on regular follow up. Exemestane tab for 5 years and radiotherapy (50 gray in 25 fractions) was applying on right breast. One year and half after diagnosed primary breast cancer, patient complained from severe diarrhea 8 times per day watery contents, vomiting and suffered from lower abdominal pain. PET scan for whole body in October 2014 was done; the results showed ascending colon is highly suspicious for malignancy and moderately hypermetabolic left adrenal mass. Subtotal colectomy surgery was done, the pathology report of biopsy revealed low grade malignant neuroendocrine neoplastic lesion stage of T3N1Mo. Physician prescribed octreotide acetate $20 \mathrm{mg}$ I. M monthly due to neuroendocrine lesion. In January 2016, cancer recurrence in the same right breast, IHC revealed ER+, PR-, Her $2+$, physician decided to change exemestane to fulvestrant $250 \mathrm{mg} \mathrm{s}$. c for 6 cycles. Radiation therapy was applied 20 gray in 10 fractions on scar. In May 2016, CAP-CT scan result revealed two enlarged left axillary L.N and left soft tissue density adrenal mass $(3.0 \times 2.3)$. Excisional lymph node was done which revealed IDC, ER+, PR-, Her 2+. Physician decided to discontinue fulvestrant and switch to exemestane $20 \mathrm{mg}$ monthly. CAP-CT was done in Feb 2017; single left axillary L.N $1 \mathrm{~cm}$, small hypodense lesion (spleen $4 \mathrm{~cm}$ ), left
\end{abstract}


adrenal lesion $(2.2 \times 2.6 \mathrm{~cm})$ and osteolytic lesion were noted in iliac areas, so the physician considered those results a metastatic area from breast and prescribed lapatinib 84 tablets. Patient now onoctreotide acetate $20 \mathrm{mg}$ I. M, trastuzumab $440 \mathrm{~m}$, exemestane and lapatinib tablets) monthly, zoledronic acid $4 \mathrm{mg}$ q 3 months, patient now still on follow up with a good condition. Conclusion: Breast cancer metastatic to left adrenal gland which is extremely rare especially when they originate from IDCs. The present case is the seventh breast cancer metastatic to the adrenal gland in the literature up to our search. Neuroendocrine tumor was happened in colon after one year and half which was a rare second primary malignancy (SPM) among female breast cancer.

\section{Keywords}

Breast Cancer, Neuroendocrine Tumor, Gene, Adrenal Gland, Second Primary

\section{Introduction}

Breast cancer is the most common cancer among female in the world, it is approximately around $25 \%$ of all cancers in 2012 [1]. Invasive ductal carcinoma (IDC) is the most common histological type of breast cancer, accounting for up to $85 \%$ of all breast cancers [2]. The most common organs can invasive ductal carcinoma (IDC) easily metastasis are lungs, bone, brain and liver, but less frequently it can also spread to ovaries, spleen, pancreas and kidneys [3]. Metastasis to adrenal glands from breast tumors is rare especially when they originate from IDCs [4].

Neuroendocrine tumors (NET) of colon are increasingly diagnosed in the US, with an annual incidence of 1 per 100,000 [5]. Most common site is GI tract accounting for more than $60 \%$ of NET [6]. Approximately $50 \%$ of patients are asymptomatic, but sometimes patients suffer from rectal bleeding, pain, and diarrhea, even during the metastatic stage [7].

We reported a rare case of metastatic invasive breast ductal carcinoma to the left adrenal gland in addition second primary cancer in colon which was called low grade malignant neuroendocrine neoplastic lesion. Up to our search, this case is the seventh case to be presented in the literature that was described breast cancer metastatic to adrenal gland. The present study was approved by the Ethics Committee of Al-basheer Hospital and ministry of health after getting the acceptance from head of oncology department and the written informed consent was provided by the patient and her daughter.

\section{Case Report}

A 68 years old female, was diagnosed as a case of right breast cancer in 2013; grade II/III according to Bloom Richardson grading. She had negative medical and family history regarding breast cancer. Her first menstrual history was at age 
15. She became pregnant at 20 years old. The patient has 12 children all of them were breast feeding. Modify radical mastectomy (MRM) was done and the pathology report showed that the histopathology type of tumor was (IDC), moderately differential (comedo type), tumor size $7 \mathrm{~cm}$ in greatest dimention and lumph node involvement by tumor 16/19. Finally stage T3N3M0. Immunohisrochemisty (IHC) study for hormonal receptors findings revealed a tumor with ER positive, PR positive and Her 2 positive (Triple positive). Patient refused to treat by chemotherapy but she accepted to treat by hormonal and target therapy.

Patient was given trastuzumab (first dose $8 \mathrm{mg} / \mathrm{kg}$, followed by $6 \mathrm{mg} / \mathrm{kg}$ every three weeks) i. v 16 cycles for the period of one year after which she went on regular follow up. Exemestane tab was prescribed for 5 years and 50 gray in 25 fractions radiation therapy was applying on right breast.

After one year and half from primary diagnosis of breast cancer; patient complained from severe diarrhea 8 times per day watery contents, vomiting and suffered from lower abdominal pain. Physician prescribed drugs to alleviate the pain and to return bowel movement to the normal but patient still suffered from diarrhea despite of drug administration. So physician decided to do PET scan for whole body in October 2014, the result showed hypermetabolic colonic wall thickening (ascending colon) is highly suspicious for malignancy and moderately hypermetabolic left adrenal mass.

In November 2014, patient was done colonoscopy which revealed moderate differentiation adenocarcinoma. Subtotal colectomy surgery was done, the pathology report of biopsy (ileim, cecum, ascending colon, transverse colon \& right hemicolectomy revealed low grade malignant neuroendocrine neoplastic lesion (well differentiated), and stage of T3N1Mo. Special immunostains was done and results were as follows: NSE, chromogranin A, S-100 were all positive for neoplastic cells, while CD36, vimentin V9 were positive in fibrovascular areas surrounding neoplastic cell. Physician prescribed octreotide acetate $20 \mathrm{mg} \mathrm{I}$. M monthly due to neuroendocrine lesion.

In May 2015, Chest, abdomen and pelvic-CT showed abnormal finding soft tissue density left adrenal mass lesion $(2.2 \times 1.9 \mathrm{~cm})$ see Picture $(1)$.

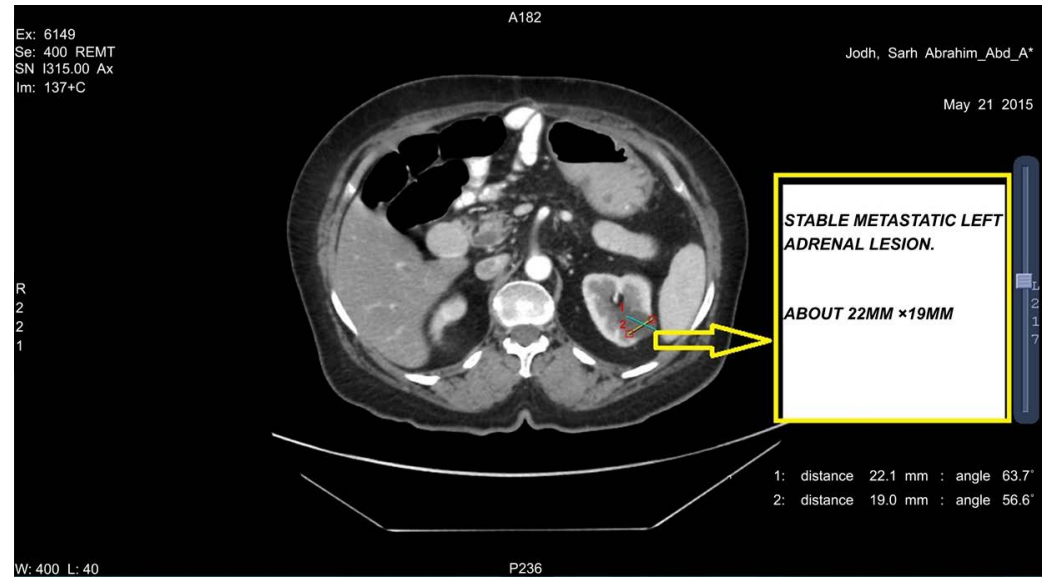

Picture 1. 1:Stable left adrenal gland which showed in CAP on 21/May /2015. 
In January 2016, patient has two nodules at the site of scare, after removing them, the histopathology report showed (recurrence in the same right breast), IDC, grade II moderately differentiated, IHC revealed ER+, PR-, Her 2+, physician decided to change exemestane to fulvestrant $250 \mathrm{mg} \mathrm{s}$. c for 6 cycles and radiation therapy was applied 20 gray in 10 fractions on scar. In may 2016, mammogram of left breast showed cluster group of macrocalcification in UOQ and need for further evaluation, CAP-CT scan confirmed two enlarged left axillary L. $\mathrm{N}$ and left soft tissue density adrenal mass $(3.0 \times 2.3)$ see Picture $(2)$. Excisional lymph node revealed IDC, ER+, PR-, Her 2+ and the right breast mass re-excision showed foci of giant cell.

Physician decided to discontinue fulvestrant, switch to exemestane $20 \mathrm{mg}$ monthly. Pt still on trastuzumab $440 \mathrm{mg}$ and octreotide acetate $20 \mathrm{mg}$ I. M monthly.

In august 2016, patient has osteopenia according to bone densitometry report, physician prescribed zoledronic acid $4 \mathrm{mg}$ i. v every 3 months. Patient now on follow up, left breast ultrasound showed ill defined hypoechoic lesion with post acoustic shadow with extension to the nipple about $3 \times 1.2 \mathrm{~cm}$ retroareolar. The result of CAP-CT in Feb 2017; confirmed single left axillary L. N $1 \mathrm{~cm}$, small hypodense lesion (spleen $4 \mathrm{~cm}$ ), left adrenal lesion $(2.2 \times 2.6 \mathrm{~cm})$ see Picture (3)

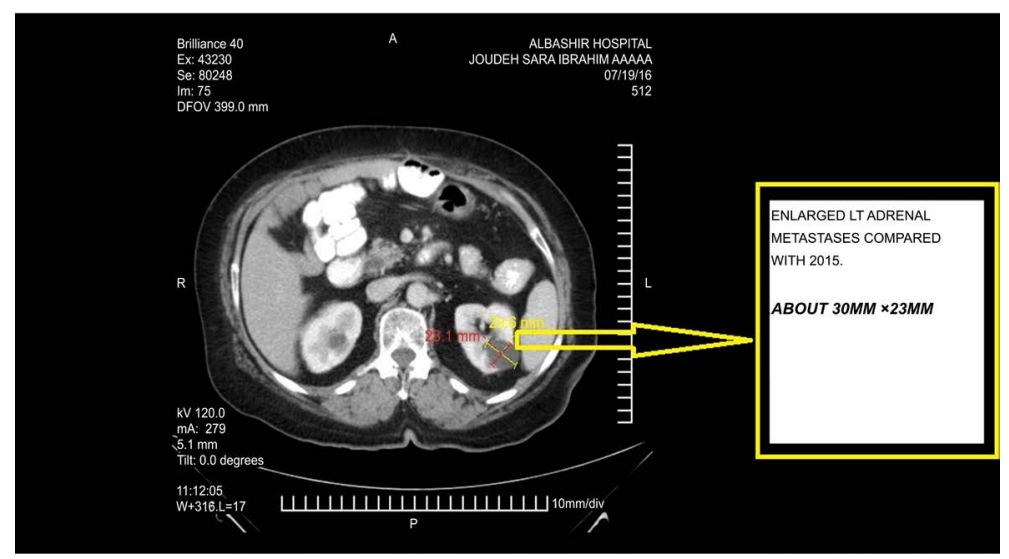

Picture 2. CAP-CT on 19/July/ /2016 showed enlarged left adrenal gland metastasis.

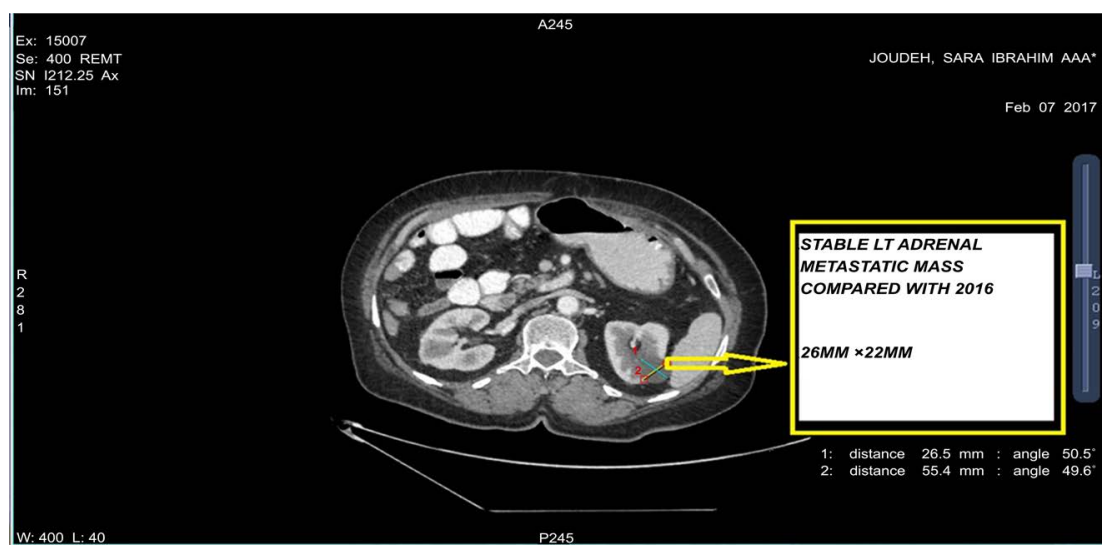

Picture 3. CAP-CT on 7/Feb./2017 showed stable left adrenal gland metastasis lesion. 
and osteolytic lesion was noted in iliac areas, so the physician consider those results a metastatic areas from breast and prescribed lapatinib tabs.

Patient now on octreotide acetate $20 \mathrm{mg}$ I. M, trastuzumab $440 \mathrm{mg}$, exemestane and lapatinib tablets monthly, zoledronic acid $4 \mathrm{mg}$ q 3 months, patient now still on follow up with a good condition.

\section{Discussion}

Patients with right sided breast cancer are more likely to have a relative with breast cancer.

Right Breast cancer is usually happened in younger women have a genetic and history relation, more negative receptors with smaller tumor size and metastasis to the bone early and more aggressive behaviors compared to the left side breast cancer [8]. but in this case; patient has 68 years old and has no any cancer history of her family, tumor size $7 \mathrm{~cm}$ and metastatic to adrenal gland first which is non consist with literature review but there is a congruent with aggressiveness of disease comparing with No sheen Fatima retrospective study in 2013.

According to Local Breast Cancer Recurrence; the risk of local recurrence decreased with event-free time. Overall, it was $1 \%$ after 3 event-free years and 3\% within 5 years after diagnosis, it differed by subtype, with ER-positive, PR-positive, HER2-negative breast cancer with the lowest risk and triple-negative with the highest risk. The risk was $2.2 \%$ with triple and with ER+, PR-, HER 2- was 2.4\% [9]. Comparing with our case; the recurrence of disease was happened after 3 years in the same right breast with translation of receptors, in addition after one year from the local recurrence, the cancer spread to left breast with aggressive behavior may be due to rejection chemotherapy treatment.

In addition, breast cancer metastatic to left adrenal gland which is extremely rare especially when they originate from IDCs versus ILC which is associated with adrenal gland metastasis [10]. Up to our search, only 6 cases have been reported previously in the literature (Table 1).

The present case is the newest and the seventh in the literature. The adrenal gland metastases are asymptomatic in the majority of patients. It is difficult to distinguish between primary adrenal gland tumor and metastatic adrenal gland, to identify the diagnosis, the physician must do CT scan with pathological examination [12] and to confirm the diagnosis, Each patient who has a history of malignance and mass $>2 \mathrm{~cm}$ in diameter in adrenal gland must be highly considered as a possible case of adrenal gland metastasis [17]. The patient in the present case was asymptomatic with no abdominal pain or other problems, all of laboratory test within normal range, however; she had a history of right and left breast carcinoma and a CAP-CT revealed left soft tissue density adrenal mass $(3.0 \times 2.3)$ which was considered as a possible metastasis.

According to second primary cancer in our case, neuroendocrine tumor was happened in colon after one year and half from primary diagnosis of breast cancer which was a rare second primary malignancy (SPM) among female breast 
Table 1. Reported cases of metastatic adrenal gland from Invasive ductal carcinoma.

\begin{tabular}{|c|c|c|c|c|c|c|c|}
\hline References & No of case & Authors & years & Location & Tumor size & Sign \& symptoms & Immunohistrochemistry \\
\hline [11] & 2 & Yoshitomi et al., 2012 & 46 & Right adrenal gland & NA & Asymptomatic & E-cadherin,$+ \mathrm{CK}+$ \\
\hline [12] & 1 & 1 Liu et al., 2010 & 64 & Left adrenal gland & 6 & Asymptomatic & C-erbB-2+, GCDFP-15+, \\
\hline [13] & 3 & Akhtar et al., 2012 & 45 & Left adrenal gland & 2.8 & $\begin{array}{l}\text { Pain in abdomen, } \\
\text { shortness of breath }\end{array}$ & ER,+ Pg R+, HER 2+ \\
\hline [14] & 4 & Eren et al., 2012 & 38 & Left adrenal gland & 4 & Asymptomatic & NA \\
\hline [15] & 5 & $\begin{array}{c}\text { Andjelic-Dekic N. } \\
\text { et al., } 2014\end{array}$ & 58 & Left adrenal gland & NA & Asymptomatic & HER2+ \\
\hline [16] & 6 & TAO HE. et al., 2016 & 35 & Left adrenal gland & 2.5 & Asymptomatic & $\mathrm{ER}+, \mathrm{PR}+, \mathrm{Her} 2+$ \\
\hline
\end{tabular}

cancer. Our results are in contrast to previous studies in literature. The result of cohort study conducted by Hung M-H et al. in 2016 which demonstrated the occurrence of second primary tumor was significantly higher for uterine, thyroid then bone and soft tissue cancers among female breast cancer patients [18]. In Dutch female breast cancer patients; the standardized incidence ratios (SIRs) were elevated for cancers of the esophagus, stomach, colon, rectum, lung, uterus, ovary, kidney, and bladder cancers, and for soft tissue sarcomas (STS), melanoma, non-Hodgkin's lymphoma, and acute myeloid leukemia (AML) respectively, Among patients age 50 years and older, radiotherapy was associated with raised STS risk, chemotherapy with increased risks of melanoma, uterine cancer, and AML; and hormonal therapy with uterine cancer [19].

Up to our search in pubmed, ELM data base, there was a rare case of Multiple endocrine neoplasia type 1 (MEN1) which contains carcinoid tumors associated with breast cancer with the MEN1 gene mutation in a 45-year-old female was diagnosed with breast cancer. A germ-line MEN1 gene mutation was detected and it could be assumed that MEN1 syndrome may have possibly predisposed the present patient to breast cancer [20]. But in Furuuchi and his colleagues study in 2000; APC gene mutations presented in 57\% of colorectal cancers and in $18 \%$ of breast cancers. APC mutations were observed at a significantly high frequency in advanced stages of primary breast cancers (TNM classification, $P<$ 0.05) [21]. So there is APC gene mutation association between breast and colorectal cancers. However; additional case reports and studies are required to illustrate gene mutation and gene association between breast cancer and neuroendocrine tumors.

\section{Conclusion}

The present case is considered as a rare case of right breast cancer in patient has 68 years old and has no any cancer history of her family, with large tumor size 7 $\mathrm{cm}$ and metastatic to adrenal gland first which does not consist with literature review. Breast cancer metastatic to left adrenal gland is extremely rare especially when they originate from IDCs. The present case is the seventh breast cancer metastatic to the adrenal gland in the literature up to our search. Neuroendo- 
crine tumor happened in colon after one year and half which was a rare second primary malignancy (SPM) among female breast cancers. However, additional case reports are required to illustrate gene mutation and gene association between breast cancer and neuroendocrine tumors.

\section{Conflicts of Interest}

Conflict of interest relevant to this case study was not reported.

\section{Acknowledgements}

We would like to thank the daughter of patient for her participation and corporation to discuss this case study, she give us all radiology pictures with original reports and helped us by answering for all question and about her status with cooperative manner.

\section{References}

[1] Ferlay, J, Soerjomataram, I., Dikshit, R., et al. (2014) Cancer Incidence and Mortality Worldwide: Sources, Methods and Major Patterns in GLOBOCAN 2012. International Journal of Cancer, 136, E359-386. https://doi.org/10.1002/ijc.29210

[2] Toikkanen, S., Pylkkänen, L. and Joensuu, H. (1997) Invasive Lobular Carcinoma of the Breast Has Better Short- and Long-Term Survival than Invasive Ductal Carcinoma. British Journal of Cancer, 76, 1234-1240. https://doi.org/10.1038/bjc.1997.540

[3] Lacroix, M. (2006) Significance, Detection and Markers of Disseminated Breast Cancer Cells. Endocrine-Related Cancer. Endocrine Related Cancer, 13, 1033-1067. https://doi.org/10.1677/ERC-06-0001

[4] Barros, N.A., Ferreira, A., Rocha, M.J., et al. (2015) Unusual Breast Cancer Metastasis. BMJ Case Reports, 2015, 43-45. https://doi.org/10.7314/APJCP.2013.14.1.43

[5] Yao, J.C., Phan, A.T., Chang, D.Z., et al. (2008) Efficacy of RAD001 Everolimus and Octreotide LAR in Advanced Low- to Intermediate-Grade Neuroendocrine Tumors: Results of a Phase II Study. Journal of Clinical Oncology, 26, 4311-4318. https://doi.org/10.1200/JCO.2008.16.7858

[6] Hallet, J., Law, C.H., Cukier, M., et al. (2015) Exploring the Rising Incidence of Neuroendocrine Tumors: A Population-Based Analysis of Epidemiology, Metastatic Presentation, and Outcomes. Cancer, 121, 589-597. https://doi.org/10.1002/cncr.29099

[7] Wang, A.Y. and Ahmad, N.A. (2006) Rectal Carcinoids. Current Opinion in Gastroenterology, 22, 529-535. https://doi.org/10.1097/01.mog.0000239868.27328.1d

[8] Fatima, N., Zaman, M.U., Maqbool, A., et al. (2013) Lower Incidence but More Aggressive Behavior of Right Sided Breast Cancer in Pakistani Women: Does Right Deserve More Respect? Asian Pacific Journal of Cancer Prevention, 14, 43-45.

[9] O’Rourkehe, K. (2016) Study Refines the Risk for Breast Cancer Recurrence. Medscape, Society of Surgical Oncology (SSO) 2016 Cancer Symposium: Abstract 4.

[10] Bumpers, H.L., Hassett, J.M., Penetrante, R.B., et al. (1993) Endocrine Organ Metastases in Subjects with Lobular Carcinoma of the Breast. Archives of Surgery, 128, 1344-1347. https://doi.org/10.1001/archsurg.1993.01420240052009

[11] Yoshitomi, S. and Tsuji, H. (2012) A Case of Recurrent Breast Cancer with Solitary 
Adrenal Gland Metastasis Treated with Surgery and Endocrine Therapy. Gan To Kagaku Ryoho, 39, 2074-2076.

[12] Liu, X.J., Shen, P., Wang, X.F., et al. (2010) Solitary Adrenal Gland Metastasis from Invasive Ductal Breast Cancer: An Uncommon Finding. World Journal of Surgical Oncology, 8, 7. https://doi.org/10.1186/1477-7819-8-7

[13] Eren, O.O., Ordu, C., Selcuk, N.A., et al. (2016) Bilateral Synchronous Adrenal Metastasis of Invasive Ductal Carcinoma Treated with Multimodality Therapy Including Adrenalectomy and Oophorectomy. Journal of Oncology Pharmacy Practice, 22, 157-160. https://doi.org/10.1177/1078155214551314

[14] Akhtar, K., Sherwani, R. and Kahkhashan, E. (2012) Carcinoma Breast Metastasis to the Suprarenal Gland: An Unusual Presentation. Polish Journal of Pathology, 63, 284-285. https://doi.org/10.5114/pjp.2012.32777

[15] Andjelić-Dekić, N., Božović-Spasojević, I., Milošević, S., et al. (2014) A Rare Case of Isolated Adrenal Gland Metastasis of Invasive Ductal Breast Carcinoma. Srpski Arhiv Za Celokupno Lekarstvo, 142, 597-601. https://doi.org/10.2298/SARH1410597A

[16] He, T., Liu, J., Li, Y., et al. (2016) Left Adrenal Gland Metastasis of Breast Invasive Ductal Carcinoma: A Case Report. Molecular and Clinical Oncology, 4, 859-862. https://doi.org/10.3892/mco.2016.814

[17] Suzuki, H. (2006) Laparoscopic Adrenalectomy for Adrenal Gland Carcinoma and Metastases. Current Opinion in Urology, 16, 47-53. https://doi.org/10.1097/01.mou.0000193378.14694.9b

[18] Hung, M.H., Liu, C.J., Teng, C.J., et al. (2016) Risk of Second Non Breast Primary Cancer in Male and Female Breast Cancer Patients: A Population-Based Cohort Study. PLoS ONE, 11, e0148597. https://doi.org/10.1371/journal.pone.0148597

[19] Schaapveld, M., Visser, O., Louwman, M.J., et al. (2008) Risk of New Primary Nonbreast Cancers after Breast Cancer Treatment: A Dutch Population-Based Study. Journal of Clinical Oncology, 26, 1239-1246.

[20] Jeong, Y.J., Oh, H.K. and Bong, J.G. (2014) Multiple Endocrine Neoplasia Type 1 Associated with Breast Cancer: A Case Report and Review of the Literature. Oncology Letters, 8, 230-234. https://doi.org/10.3892/ol.2014.2144

[21] Furuuchi, K., Tada, M., Yamada, H., et al. (2000) Somatic Mutations of the APC Gene in Primary Breast Cancers. American Journal of Pathology, 156, 1997-2005. https://doi.org/10.1016/S0002-9440(10)65072-9 